\title{
Timeliness of Outpatient Follow-up: An Evidence-Based Approach for Planning After Hospital Discharge
}

\author{
Carlos Jackson, $P b D^{1}$ \\ Mobammad Shabsabebi, MD, MBA² \\ Tiffany Wedlake, MD, MPH \\ C. Annette DuBard, MD, MPH \\ 'Community Care of North Carolina, \\ Raleigh, North Carolina \\ ${ }^{2}$ Duke Family Medicine, Duke University \\ Medical Center, Durham, North Carolina
}

\begin{abstract}
PURPOSE Timely outpatient follow-up has been promoted as a key strategy to reduce hospital readmissions, though one-half of patients readmitted within 30 days of hospital discharge do not have follow-up before the readmission. Guidance is needed to identify the optimal timing of hospital follow-up for patients with conditions of varying complexity.
\end{abstract}

METHODS Using North Carolina Medicaid claims data for hospital-discharged patients from April 2012 through March 2013, we constructed variables indicating whether patients received follow-up visits within successive intervals and whether these patients were readmitted within 30 days. We constructed 7 clinical risk strata based on 3M Clinical Risk Groups (CRGs) and determined expected readmission rates within each CRG. We applied survival modeling to identify groups that appear to benefit from outpatient follow-up within 3, 7, 14, 21, and 30 days after discharge.

RESULTS The final study sample included 44,473 Medicaid recipients with 65,085 qualifying discharges. The benefit of early follow-up varied according to baseline readmission risk. For example, follow-up within 14 days after discharge was associated with 1.5\%-point reduction in readmissions in the lowest risk strata $(P<.001)$ and a $19.1 \%$-point reduction in the highest risk strata $(P<.001)$. Follow-up within 7 days was associated with meaningful reductions in readmission risk for patients with multiple chronic conditions and a greater than $20 \%$ baseline risk of readmission, a group that represented $24 \%$ of discharged patients.

CONCLUSIONS Most patients do not meaningfully benefit from early outpatient follow-up. Transitional care resources would be best allocated toward ensuring that highest risk patients receive follow-up within 7 days.

Ann Fam Med 2015;13:115-122. doi: 10.1370/afm.1753.

\section{INTRODUCTION}

$\mathrm{R}$ educing hospital readmissions is a current priority for the health care system. Timely outpatient follow-up is promoted as a key component of transitional care models that have been successful in reducing readmission rates, such as the Care Transitions Intervention, the Transitional Care Model, Project RED, and Better Outcomes by Optimizing Safe Transitions (BOOST). ${ }^{1-4}$ In January 2013, Medicare-implemented payment incentives for follow-up appointments within 7 and 14 days of discharge further emphasize timely follow-up after discharge as a strategy to reduce readmission. ${ }^{5,6}$ To date, however, these recommendations rely largely on expert opinion, and there has been little evidence to guide best practices for the timing of follow-up care after hospital discharge.

The focus of most research examining the impact of outpatient follow-up on preventing readmissions has been narrow, focusing on specific disease states. Numerous studies have shown that early outpatient follow-up is associated with lower readmission rates for heart failure, ${ }^{7-9}$ and similar observations have been made in studies of hospitalized patients with pediatric asthma, ${ }^{10}$ sickle cell disease, ${ }^{11}$ and chronic obstructive pulmonary disease. ${ }^{12}$ Studies of general hospitalized populations, however, have shown

\section{CORRESPONDING AUTHOR}

C. Annette DuBard, MD, MPH 2300 Rexwoods Dr Raleigh, NC 27612 adubard@n3cn.org 
mixed results, ${ }_{1}^{13-15}$ with 1 study showing no difference in 30-day readmission rates related to timing of outpatient follow-up among patients discharged from the internal medicine service at an academic medical center. ${ }^{13}$

Nationally, approximately one-half of Medicare and Medicaid beneficiaries readmitted within 30 days of a hospital discharge do not receive outpatient follow-up before the readmission. ${ }^{16,17}$ Low rates of outpatient follow-up have been similarly noted in other cohorts of patients rehospitalized for chronic conditions, ${ }^{7-12}$ suggesting that the opportunity to reduce readmissions through timely follow-up may be large and widespread. Even so, effective strategies are lacking to assure optimal follow-up for patients who stand to benefit. In the context of a statewide initiative to improve care transitions for Medicaid recipients in North Carolina, we hypothesized that risk segmentation may be a useful approach to identify patients most in need of early follow-up after hospital discharge. Our intent was to better target efforts to help patients secure and keep outpatient appointments toward those most likely to benefit. In this retrospective analysis of Medicaid claims data, we specifically wanted to test whether readmission risk is associated with the likelihood of benefit from early outpatient follow-up; furthermore, we wanted to determine the optimal time by which patients with conditions of varying clinical complexity should receive outpatient follow-up after discharge.

\section{METHODS}

\section{Setting}

Community Care of North Carolina (CCNC) is a public-private partnership that links Medicaid recipients to primary care medical homes and provides quality improvement and care management support for highrisk patients. During this analysis, more than $70 \%$ of NC Medicaid recipients were enrolled in CCNC, with more than 1,600 participating primary care practices throughout the state. This study was conducted for intervention-planning purposes to develop care coordination standards for the CCNC Transitional Care program described elsewhere. ${ }^{18,19}$

\section{Data Source}

Data for this project included statewide inpatient, outpatient, professional, and pharmacy claims paid by NC Medicaid for dates of service between January 1, 2008, and April 30, 2013.

\section{Sample Selection}

For the primary study analysis, inpatient visits were included if the patient was discharged to home during the period April 1, 2012, through March 31, 2013, excluding childbirth and newborn discharges. Patients had to be enrolled in a CCNC primary care medical home at the time of discharge and not dually enrolled in Medicare at the time of discharge. Patients were allowed to contribute more than 1 index admission during the study period.

\section{Benchmark Readmission Rates}

To determine benchmark or expected 30-day readmission rates for Medicaid patients in the primary analysis, we analyzed 5 years of NC Medicaid inpatient claims (2008-2012), including 192,616 qualifying discharges among 84,288 patients, to determine an average 30-day readmission rate by Clinical Risk Group (CRG). Developed by 3M Health Information Systems, CRGs use a hierarchical model to assign patients into 1 of 1,075 mutually exclusive clinical risk groupings based on historical claims data, using diagnosis and procedure history to assess the number and severity of chronic conditions. ${ }^{20}$ The average 30 -day readmission rate among patients in each of the 1,075 CRGs during this 5 -year period determined the expected readmission rate for study patients within the same CRG.

\section{Clinical Risk Strata}

Clinical risk strata were determined according to risk of 30-day readmission specific to a patient's CRG. We defined 7 distinct clinical risk strata by aggregating CRGs according to expected readmission rate. Patients whose CRG indicated that they did not have a moderate or dominant chronic condition comprised the no chronic condition group, with an expected 30-day readmission rate of less than $10 \%$. Patients whose CRG indicated that they had only 1 moderate or dominant chronic condition comprised the single chronic condition group, which also had an expected 30-day readmission rate of less than $10 \%$. Patients whose CRG indicated that they had multiple moderate or dominant chronic conditions were further stratified into 1 of 5 groups (less than 10\%, 10\%-19\%, 20\%-29\%, 30\%-39\%, and $40 \%$ or greater) based upon the 30 -day readmission rate expected for patients with their respective CRGs.

\section{Design}

The primary analysis examined time to readmission for patients who received outpatient follow-up within a specified number of days after discharge compared with those who did not, further stratified by the 7 clinical risk strata. Readmissions were defined as a return to any hospital within 30 days of a previous discharge for any reason other than childbirth. The readmission did not need to be clinically related to the initial admission, and no distinction was made between planned or unplanned readmissions. Out- 
patient follow-up was defined as an office visit with any primary care or specialist physician or to a federally qualified health center, as indicated by a professional claim with a Current Procedural Terminology (CPT) code for evaluation and management or Health Care Common Procedure Coding System (HCPCS) code for all-inclusive clinic visit.

\section{Statistical Analysis}

For the survival analysis examining time to readmission, we constructed 5 intervention variables corresponding to whether the patient received a first follow-up encounter within 3, 7, 14,21 , and 30 days after discharge. A patient who had a follow-up visit on day 4 would be coded as not having had follow-up within 3 days, but would have been included in the intervention group for the 7-, 14-, 21-, and 30-day variables. A patient who received follow-up at day 30 would have been included in the comparison arm for each group with earlier follow-up intervals. Patients were censored at death or when readmitted to the hospital. Group differences were tested using the Wilcoxon-Gehan statistic. For patients with multiple hospitalizations during the study period, each qualifying discharge was included in the survival analysis as a new index event. Finally, we conducted several sensitivity analyses, repeating the analyses within specific subgroups, to examine whether our findings held true when controlling for patient-level characteristics that may be correlated with both the intervention (outpatient follow-up) and the outcome (30-day readmissions).

\section{RESULTS}

The study sample included 44,473 unique Medicaid recipients with 65,085 qualifying discharges. Most patients only had 1 discharge, with $95 \%$ having 3 or fewer qualifying discharges during the year. Discharges occurred from 114 hospitals statewide. Patients resided in all of North Carolina's 100 counties and were enrolled in 1,576 primary care medical homes. In the total study sample, $49 \%$ were younger than 21 years, $55.3 \%$ were female, and $49.6 \%$ were white (Table 1 ).

Thirty-day survival rates from hospital readmission are displayed in Table 2 by risk strata and whether the patient received follow-up within successively longer intervals after discharge from the hospital. Earlier outpatient follow-up was associated with statistically significant survival from readmission within every risk stratum by 14 days after discharge. The magnitude of the effect of earlier follow-up, however, was far more pronounced in higher risk strata. For example, among patients with no chronic conditions, those who had follow-up within 14 days had 1.5 percentage point higher rates of readmission survival than those who did not $(P<.001)$. Among patients with multiple chronic conditions in the 3 highest risk strata, that difference difference ranged from 12.0 to 19.1 percentage points $(P<.001)$. Of note, only $51 \%$ of these patients received follow-up within 14 days, a rate similar to lower risk patients (50\%).

Readmission survival curves for each clinical risk stratum are illustrated in Figures 1 and 2, which compare those who received follow-up within 7 days with those who did not. Although there is minimal apparent benefit of early follow-up for the lowest risk strata, separation is clearly evident within the higher risk strata (patients with multiple chronic conditions and a greater than $20 \%$ risk of readmission).

Figure 3 illustrates the differential impact across successive risk strata as a function of when follow-up occurred. Each point on this figure represents the results of a survival analysis. Each of the 7 risk strata was run through 5 different analyses, for a total of 35 survival analyses. We set a threshold of $5 \%$ as a clinically meaningful increase in readmission risk. The top 3 highest risk strata cross this threshold by 7 days, with the next 2 strata crossing at approximately 14 and 21 days, and the remaining lowest risk strata approaching $5 \%$ by day 30 .

\section{Sensitivity Analyses}

When limiting our analysis to include adults only, the results were not meaningfully different (incremental 
difference in readmission rates for adults receiving follow-up within 7 days: $-1.7 \%, 0.3 \%, 0.8 \%, 1.7 \%$, $7.5 \%, 6.9 \%$, and $7.8 \%$ for each of the respective strata). Additionally, when excluding behavioral health admissions, the same trends persisted (incremental difference in readmission rates: $-1.7 \%, 1.0 \%, 0.8 \%, 1.7 \%, 8.0 \%$,
$7.6 \%$, and $7.5 \%)$. Analyses by sex and race did not change these patterns.

Because some patients, particularly in the highest risk strata, may have contributed several observations to the analysis (ie, they were admitted several times during the study year), we separately examined only the first discharge during the year for each person. Resulting patterns were similar, though the incremental benefit was slightly lower than what was observed in the full sample (incremental difference in readmission rates for patients receiving follow-up within 7 days: $0.8 \%, 0.7 \%$, $0.6 \%, 1.0 \%, 4.2 \%, 4.5 \%$, and $6.4 \%$ for each of the respective strata).

\section{DISCUSSION}

This study shows that the benefit of early outpatient follow-up after hospital discharge is variable according to the patient's clinical complexity. It provides an evidence-based mechanism to determine the optimal interval for hospital follow-up based on patient risk segmentation. Although follow-up within 7 days was associated with substantially lower readmission rates among patients with highest clinical complexity and highest underlying risk of readmission, most patients do not appear to benefit from very early follow-up. Among patients with no or just 1 chronic or acute condition (representing $38 \%$ of discharges in this Medicaid population), readmissions were uncommon and negligibly affected by the timing of outpatient follow-up for up to 30 days.

Even though it may be clinically intuitive that higher risk patients need earlier follow-up, common models for improving transitional care have not emphasized risk segmentation to inform the timing of the follow-up appointment, and there has been little evidence to date upon which to base such guidance. Several studies have found improved patient outcomes and reductions in hospital use through improved care transitions in which

a Number of index discharges. 


\section{Figure 1. Time to readmission for patients who did vs did not receive outpatient follow-up within} 7 days of discharge: lower risk strata.

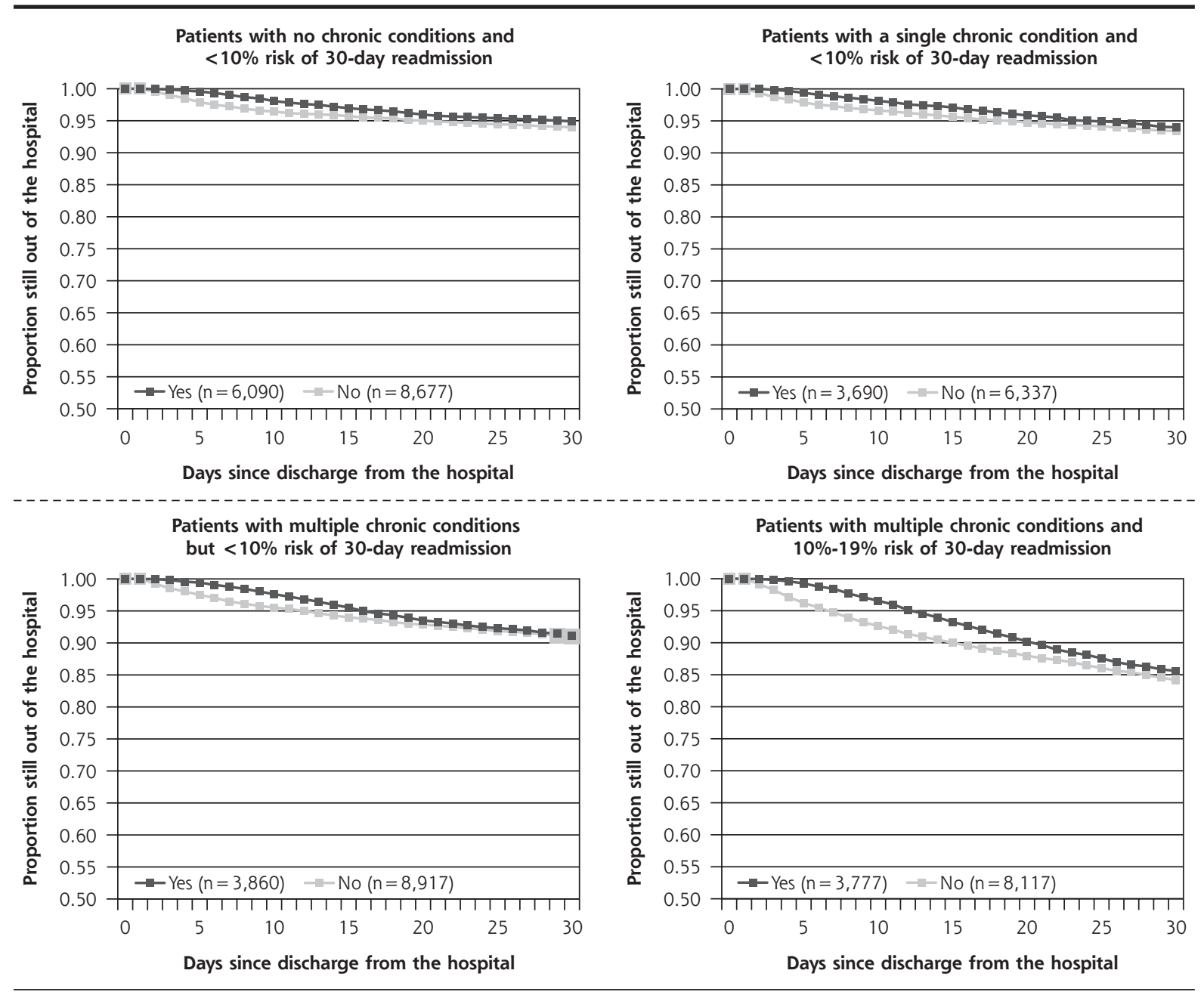

Note: Rates vary according to the patient's underlying clinical risk. Each stratum represents patients in clinical risk groups based on $3 \mathrm{M}$ Health Information System's Clinical Risk Groups and accompanying baseline risk of a 30-day readmission.

timely outpatient follow-up is considered a core component, but resource requirements have presented a barrier to widespread implementation of successful programs. To gain large-scale adoption, future transitional care models need to identify those at highest need for specific transitional care services to allow for optimal resource allocation.

Patients with multiple complex chronic conditions, who account for the great majority of readmissions among Medicaid and Medicare recipients nationally, ${ }_{1}{ }^{21}$ stand to benefit considerably from timely follow-up. For those whose readmission risk exceeds $20 \%$, our analysis suggests that 1 readmission may be prevented for every 5 patients who receive outpatient follow-up within 14 days. These patients are characterized by having 3 or more chronic conditions, often including advanced coronary artery disease, chronic obstructive pulmonary disease, chronic renal failure, congestive heart failure, diabetes, ischemic vascular disease, or a history of organ transplant, dialysis, or total parenteral nutrition. In this statewide Medicaid population, however, only $51 \%$ of patients in high-risk categories received follow-up within 14 days, and for every highrisk patient who did not receive follow-up, there was a low-risk patient who did. Healthier patients and those with greater social support or self-management skills may be better equipped to secure and attend an earlier follow-up appointment, but potentially at the cost of delaying care for those with more complex needs.

Secondary data analyses have potential biases that may limit these conclusions. Claims data provide a limited view of clinical complexity in that they record information necessary for reimbursement but not all information relevant to care decisions. Claims data 
Figure 2. Time to readmission for patients who did vs did not receive outpatient follow-up within 7 days of discharge: higher risk strata.

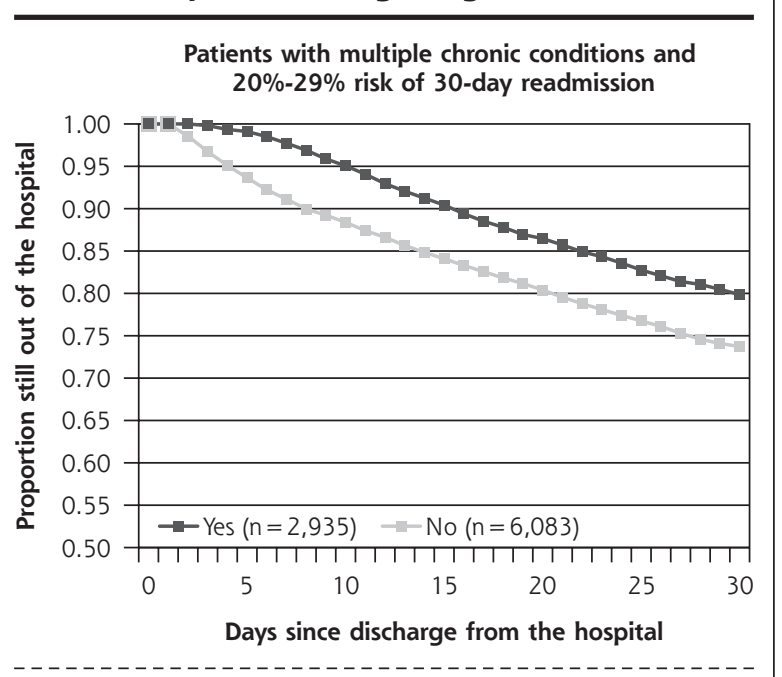

Patients with multiple chronic conditions and $30 \%-39 \%$ risk of 30 -day readmission

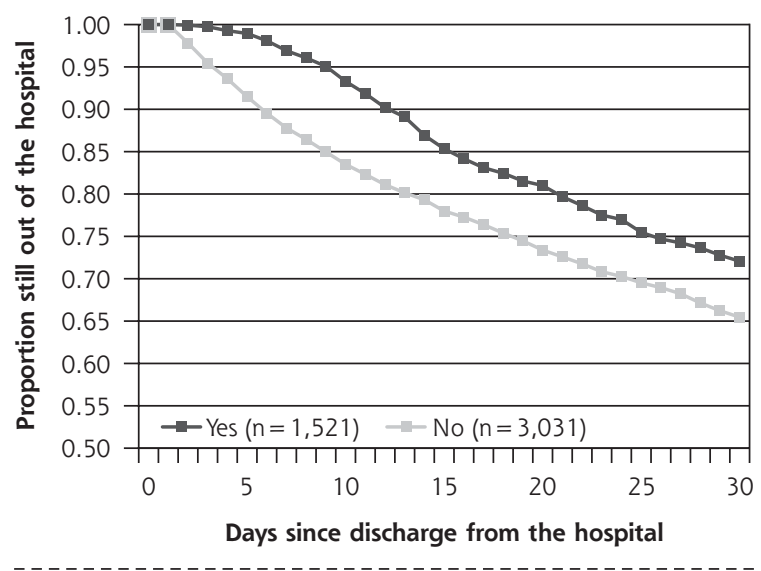

Patients with multiple chronic conditions and $\geq 40 \%$ risk of 30 -day readmission

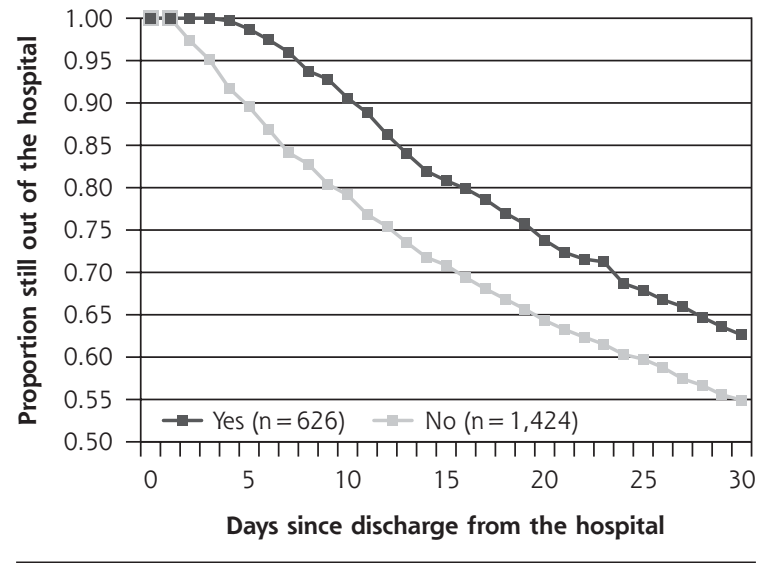

Note: Rates vary according to the patient's underlying clinical risk. Each stratum represents patients in clinical risk groups based on $3 \mathrm{M}$ Health Information System's Clinical Risk Groups and accompanying baseline risk of a 30-day readmission. also lack important predictors of readmission risk, such as functional status, health literacy, social complexity, and family or caregiver support. This study was observational, and observed associations between timeliness of outpatient follow-up and readmission occurrence do not establish a causal relationship. In addition, findings were based on data from a single state and limited to Medicaid recipients, who may be at higher risk than the general population. The study has several strengths, however, including the use of a large cohort spanning a diverse geography and multiple provider systems, as well as visibility into complete diagnosis, procedure, and medication use history, which allowed for granular examination of associations within multiple strata of clinical risk. Because patients were not randomly assigned to receive follow-up, it is possible that patients who successfully received timelier follow-up are different in unobservable ways, even when compared with patients within the same clinical risk cohort. We cannot know for certain whether having received timelier follow-up would have actually averted readmissions. Even so, this study (1) highlights the potential opportunity for improving readmissions by ensuring that patients who need timelier follow-up actually receive it, and (2) provides evidence that a significant proportion of hospitalized patients do not appear to benefit from follow-up any sooner than 30 days after hospital discharge.

In current practice, one-size-fits-all discharge protocols may be determining a follow-up time frame more than evidence-based decision making or clinical need. Changing reimbursement policies are likely to further influence transitional care processes. For example, the 2 Medicare transitional care management codes require 7-day follow-up for high complexity and 14-day follow-up for moderate complexity transitions. These codes create a financial incentive for physicians to see as many discharged Medicare patients as possible within the 7-day window. In so doing, there is the potential to positively affect after-discharge care for the Medicare population, but it risks creating an environment where patients of other payers are crowded out. As providers increasingly enter into accountable care arrangements or bundled payment structures for the period after hospital discharge, incentives will be greater for intelligently targeted resource allocation to optimize benefit across the population.

Further research is needed to determine how best to operationalize the practical application of risk segmentation to guide the timing of outpatient follow-up, which may include point-of-care decision support for physicians and personnel involved in discharge planning and scheduling in the hospital and outpatient practice. Risk segmentation may provide further value 


\section{Figure 3. Relationship between receipt of outpatient follow-up and risk of readmission.}

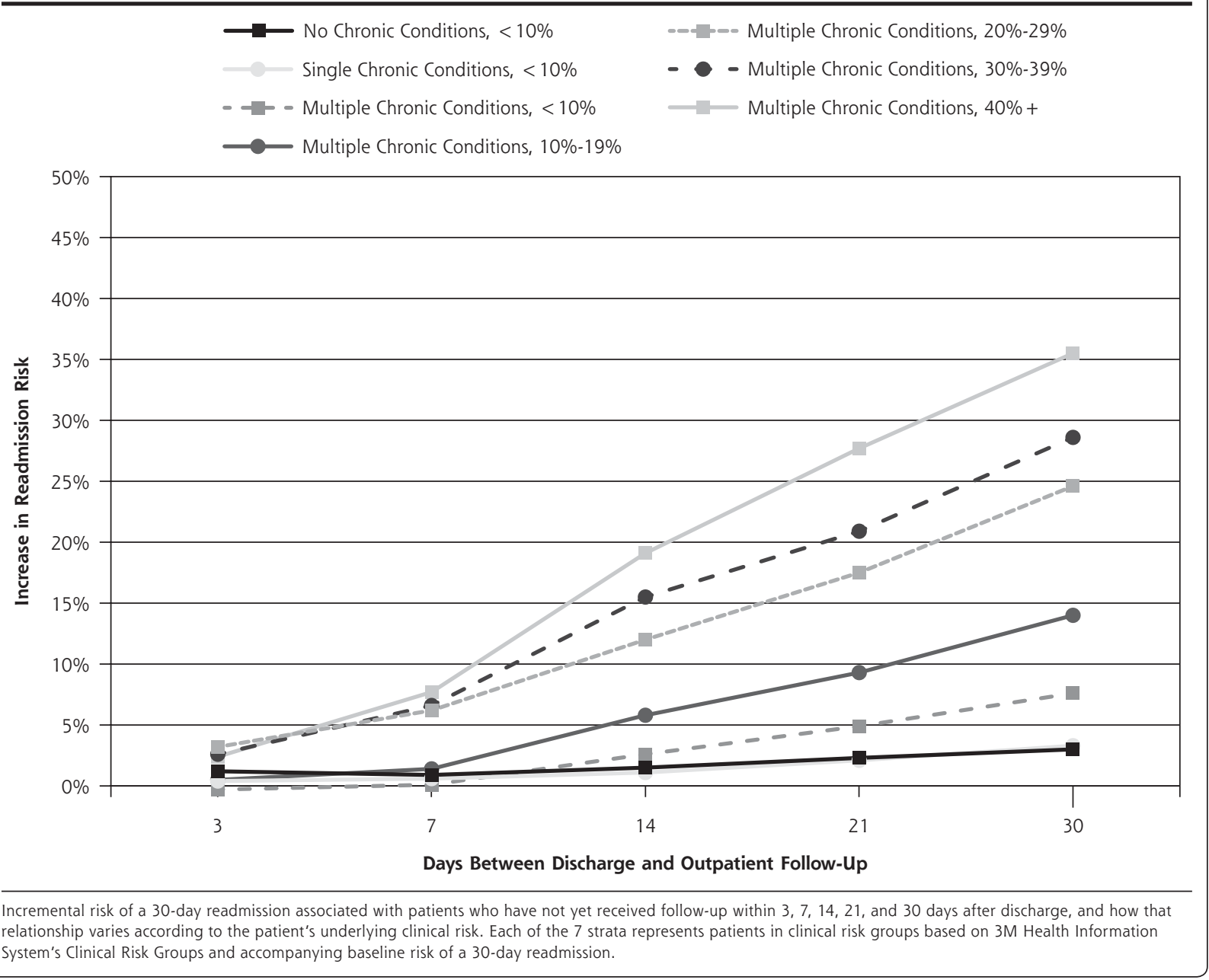

to inform optimal strategies for alternative models of follow-up contact with patients after discharge, such as nurse or pharmacist contact or other team-based approaches. Importantly, effective implementation will require sharing risk segmentation strategies and coordinating processes between inpatient and outpatient care settings. Although our analysis used historical claims data to determine a patient's readmission risk, based on the number and complexity of chronic conditions, alternative methodologies for risk segmentation to predict benefit of early follow-up should be developed and tested to accommodate the varying degrees of available data and information system capacities in today's health care environment. Such models will need to be readily incorporated into the workflows of physicians and care team members to reduce barriers to adoption and yield greatest benefit for patients and populations.

To read or post commentaries in response to this article, see it online at http://www.annfammed.org/content/13/2/115.
Key words: hospital readmissions; chronic disease; nursing care management; primary care; continuity of care

Submitted July 29, 2014; submitted, revised, November 14, 2014; accepted December 3, 2014.

Funding support: This work was partially funded by the North Carolina Healthcare Quality Alliance, and the NC Department of Health and Human Services, Office of Rural health and Community Care.

Acknowledgments: The authors would like to thank Julian Thomas and Yiwen Deng for their help with the data analysis.

\section{REFERENCES}

1. Parry C, Min SJ, Chugh A, Chalmers S, Coleman EA. Further application of the care transitions intervention: results of a randomized controlled trial conducted in a fee-for-service setting. Home Health Care Serv Q. 2009;28(2-3):84-99.

2. Naylor MD, Bowles KH, McCauley KM, et al. High-value transitional care: translation of research into practice. J Eval Clin Pract. ISSN 1365-2753.

3. Clancy CM. Reengineering hospital discharge: a protocol to improve patient safety, reduce costs, and boost patient satisfaction. Am J Med Qual. 2009;24(4):344-346. 
4. Coleman E, Williams MV. BOOSTing the hospital discharge. J Hosp Med. 2009;4(4)209-210.

5. James J. Health Policy Brief: Medicare Hospital Readmissions Reduction Program. Health Affairs (Millwood). http://www.healthaffairs.org/healthpolicybriefs/brief.php?brief_id $=102$. Published Nov 2013.

6. Medicare Learning Network. Transitional Care Management Services. ICN 908628. Centers for Medicare \& Medicaid Services, Department of Health and Human Services. http://www.cms.gov/ Outreach-and-Education/Medicare-Learning-Network-MLN/MLNProducts/Downloads/Transitional-Care-Management-Services-FactSheet-ICN908628.pdf. Published Jun 2013.

7. Ryan J, Kang S, Dolacky S, Ingrassia J, Ganeshan R. Change in readmissions and follow-up visits as part of a heart failure readmission quality improvement initiative. Am J Med. 2013;126(11):989-994.

8. Muus KJ, Knudson A, Klug MG, Gokun J, Sarrazin M, Kaboli P. Effect of post-discharge follow-up care on re-admissions among US veterans with congestive heart failure: a rural-urban comparison. Rur Rem Health. 2010;10(2):1447.

9. Hernandez AF, Greiner MA, Fonarow GC, et al. Relationship between early physician follow-up and 30-day readmission among Medicare beneficiaries hospitalized for heart failure. JAMA. 2010;303(17):1716-1722.

10. Auger KA, Kahn RS, Davis MM, Beck AF, Simmons JM. Medical home quality and readmission risk for hospitalized children hospitalized with asthma exacerbations. Pediatrics. 2013;131(1):64-70.

11. Leschke J, Panepinto JA, Nimmer M, Hoffmann RG, Yan K, Brousseau DC. Outpatient follow-up and rehospitalizations for sickle cell disease patients. Pediatr Blood Cancer. 2012;58(3):406-409.

12. Sharma G, Kuo YF, Freeman JL, Zhang DD, Goodwin JS. Outpatient follow-up visit and 30-day emergency department visit and readmission in patients hospitalized for chronic obstructive pulmonary disease. Arch Intern Med. 2010;170(18):1664-1670.
13. Kashiwagi DT, Burton MC, Kirkland LL, Cha S, Varkey P. Do timely outpatient follow-up visits decrease hospital readmission rates? Am J Med Qual. 2012;27(1):11-15.

14. Misky GJ, Wald HL, Coleman EA. Post-hospitalization transitions: Examining the effects of timing of primary care provider follow-up. J Hosp Med. 2010;5(7):392-397.

15. Hansen LO, Young RS, Hinami K, Leung A, Williams MV. Interventions to reduce 30-day rehospitalization: a systematic review. Ann Intern Med. 2011;155(8):520-528.

16. Jencks SF, Williams MV, Coleman EA. Rehospitalizations among patients in the Medicare fee-for-service program. $N$ Engl J Med. 2009;360(14):1418-1428.

17. Gilmer T, Hamblin A. Hospital readmissions among Medicaid beneficiaries with disabilities: identifying targets of opportunity. Center for Health Care Strategies, Inc. December 2010:1-10.

18. DuBard CA, Cockerham J, Jackson CT. Collaborative accountability for care transitions: the community care of North Carolina transitions program. N C Med J. 2012;73(1):34-40.

19. Jackson CT, Trygstad TK, DeWalt DA, DuBard CA. Transitional care cut hospital readmissions for North Carolina Medicaid patients with complex chronic conditions. Health Aff (Millwood). 2013;32(8):1407-1415.

20. Hughes JS, Averill RF, Eisenhandler J, et al. Clinical Risk Groups (CRGs): a classification system for risk-adjusted capitation-based payment and health care management. Med Care. 2004;42(1):81-90.

21. Centers for Medicare and Medicaid Services. Chronic Conditions Among Medicare Beneficiaries, Chart Book: 2012 Edition. Baltimore, MD: Centers for Medicare and Medicaid Services; 2012. 